\title{
Design and Analysis of a Novel Compact High Permittivity Dielectric Resonator Antenna
}

\author{
Mihai D. Rotaru and Jan K. Sykulski \\ School of Electronics and Computer Science, University of Southampton, Highfield, Southampton, SO17 1BJ, U.K.
}

\begin{abstract}
A very compact and efficient dielectric resonator antenna is proposed and investigated. The novel structure is analyzed using two numerical methods and design guidelines are established. Moreover, it is shown that — by using very high permittivity materials and appropriate design of the resonator and its feeding structure-wide frequency coverage is possible even at cellular frequencies. Simulation results are reported showing the effectiveness of the proposed antenna structure.
\end{abstract}

Index Terms-Dielectric resonator antennas, numerical simulation, very high permittivity.

\section{INTRODUCTION}

D IELECTRIC resonator antennas (DRA) have been exploited in the past at high frequencies (microwave and millimeter-wave range) due to their particular advantages over their metal structure counterparts, such as high efficiency, small size and a simple feeding arrangement [1], [2]. Because of these advantages, an implementation at cellular frequencies has been explored more recently. One of the main drawbacks of most of the reported designs, however, is the relatively large size of the resonator necessary to achieve an efficient antenna at these frequencies [3]. To obtain a reasonably sized antenna for such bands, a very high dielectric constant is required, which in turn may lead to an unacceptable reduction in bandwidth. The increase of the dielectric constant affects directly the quality factor $(Q)$ of the resonator; in fact $Q$ increases as $\varepsilon_{r}^{3 / 2}$ [4] which results in very narrow band behavior. The $Q$ of the antenna can be related to the unloaded $Q$ of the resonant mode excited by the following expression:

$$
Q=4 \pi f_{0} \frac{\text { StoredEnergy }}{\text { RadiatedPower }} \propto 4 \pi f_{0}\left(\varepsilon_{r}\right)^{p}\left(\frac{\text { Volume }}{\text { Surface }}\right)^{s}
$$

where $f_{o}$ is the resonant frequency and $\varepsilon_{r}$ is the dielectric constant of the resonator, with $p>s \geq 1$. As can be seen from (1), increasing the dielectric constant improves the quality factor (which is a welcome result as far as the resonator is concerned) but reduces the bandwidth. It also follows from (1) that by minimizing the volume-to-surface ratio the quality factor can be decreased. This idea is exploited in this work; by optimizing the shape to minimize this ratio it is shown that a high dielectric constant resonator that can support two or more close resonant modes may be achieved. In normal DRA applications such close modes are usually avoided-since the radiation pattern is strongly influenced by the modes-but in contrast in many hand-held wireless applications the radiation pattern shape plays a secondary role with respect to antenna bandwidth. The bandwidth of the antenna is also influenced by the feed, hence wide band operation requires designing a feeding structure such that the appropriate modes are efficiently coupled into

Manuscript received October 07, 2008. Current version published February 19, 2009. Corresponding author: M. D. Rotaru (e-mail: mr@ecs.soton.ac.uk).

Color versions of one or more of the figures in this paper are available online at http://ieeexplore.ieee.org.

Digital Object Identifier 10.1109/TMAG.2009.2012573 the resonator. Moreover, by choosing the appropriate feed, an efficient way of tuning the antenna may be accomplished as shown later in this paper.

\section{Design And Modeling of The AntennA}

The use of DRAs at cellular frequencies in hand-held devices has been recently explored [3], [5], but for practical purposes, it is necessary to reduce the size of the antenna. One way of accomplishing the size reduction is by using a very high dielectric constant resonator. The most important parameter of a DRA is its $Q$ which is directly related to the unloaded $Q$ of the resonant mode excited. Using a very high dielectric constant will influence the $Q$ of the antenna as described by (1) which relates the unloaded $Q$ to the geometry and material properties of the resonator. The quality factor is inversely proportional to the bandwidth, hence a high $Q$ will result in a low bandwidth or, in the worst case, in no radiation at all. For example, for a cylindrical resonator in which a $\mathrm{TE}_{01 \delta}$ mode is excited, the $Q$ factor can be written as

$$
\begin{aligned}
Q= & 0.012 \varepsilon_{r}^{1.2076} \\
& \times\left[5.27\left(\frac{D}{4 t}\right)+1106.188\left(\frac{D}{4 t}\right)^{0.625} e^{-1.0272 \frac{D}{4 t}}\right]
\end{aligned}
$$

where $D$ is the resonator diameter and $t$ its thickness. This is a particular case of (1) obtained by fitting various numerical and experimental results [6]. The numerical values of $Q$ for a cylindrical resonator with a dielectric constant of 80 , for different $D / t$ ratios, were calculated and are tabulated as follows:

\begin{tabular}{|c|c|c|c|}
\hline$D / t$ & $28 / 2$ & $20.3 / 3.8$ & $16 / 6.15$ \\
\hline$Q$ & 202.4 & 818.3 & 1041.6 \\
\hline
\end{tabular}

It can be observed that for the same volume of material a cylinder with thickness comparable in size with its radius will have a much higher $Q$ compared with a cylinder which has its thickness much smaller than its radius (coin shape).

The bandwidth of the antenna can also be extended by designing a DRA such that different modes are excited and coupled by the feed. Through this approach one can accomplish a multi-pole response from the antenna structure; furthermore, if the resonating modes are close enough, a wideband is achievable.

For a cylindrical resonator on a ground plane one can calculate, and thus predict quite accurately for practical purposes, its resonant frequencies by using the cavity resonator model 


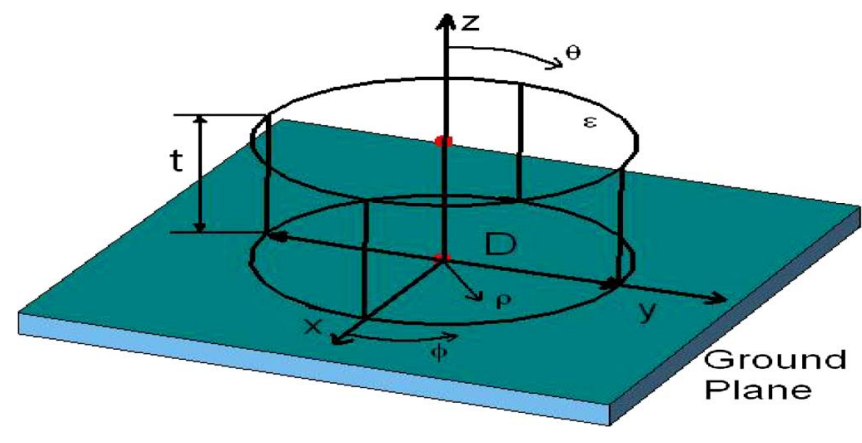

Fig. 1. Cylindrical dielectric resonator over a ground plane.

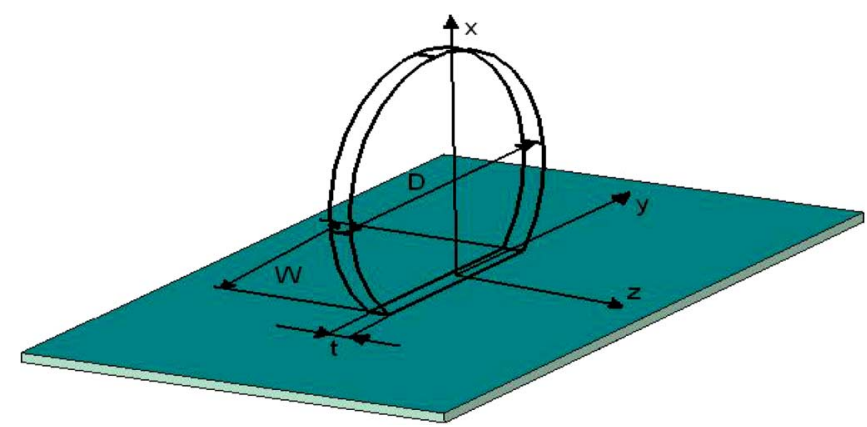

Fig. 2. Modified dielectric resonator ("C" shape) over a ground plane.

in which the outer surfaces of the cavity are considered magnetic walls [6]-[8]. In such cavities wave functions: transverse electric (TE) and transverse magnetic (TM) in $z$ direction (see Fig. 1) can be written as

$$
\begin{aligned}
& \psi_{\mathrm{TE} n p m}=J_{n}\left(\frac{2 X_{n p}}{D} \rho\right)\left\{\begin{array}{l}
\sin n \phi \\
\cos n \phi
\end{array}\right\} \sin \left[\frac{(2 m+1) \pi z}{2 t}\right] \\
& \psi_{\mathrm{TM} n p m}=J_{n}\left(\frac{2 X_{n p}^{\prime}}{D} \rho\right)\left\{\begin{array}{l}
\sin n \phi \\
\cos n \phi
\end{array}\right\} \cos \left[\frac{(2 m+1) \pi z}{2 t}\right]
\end{aligned}
$$

where $J_{n}$ is the Bessel function of the first kind, $J_{n}\left(X_{n p}\right)=0$, $J_{n}^{\prime}\left(X_{n p}^{\prime}\right)=0, n=1,2,3 \ldots, p=1,2,3 \ldots, m=0,1,2 \ldots$ Using the separation equation $k_{\rho}^{2}+k_{z}^{2}=\omega^{2} \mu \varepsilon$ one can find an expression for the resonant frequency of the $n \mathrm{pm}$ mode

$$
f_{n p m}=\frac{c}{\pi D \sqrt{\varepsilon_{r}}} \sqrt{\left\{\begin{array}{l}
X_{n p}^{2} \\
X_{n p}^{\prime 2}
\end{array}\right\}+\left[\frac{\pi D}{4 t}(2 m+1)\right]^{2}}
$$

where $D$ is the diameter of the cylindrical resonator, $t$ is its thickness, $\varepsilon_{r}$ is the resonator relative dielectric constant and $c$ is the speed of light.

Many studies have concentrated on cylindrical DRA structures like the one sketched in Fig. 1 [5], [6], [8], for which the TM and quasi-TM modes can be excited; however, little attention has been given to cylindrical DRA excited in the TE or quasi-TE modes. The main reason is that these modes cannot be excited when the circular base of the DRA is placed on the ground plane. To excite these modes, the cylindrical resonator has to be modified and one possible geometry is shown in Fig. 2.

In this new " $C$ " shaped configuration resonator the lowest resonant mode that can be excited is $\mathrm{TE}_{01 \delta}$. The resonant frequency for this mode can be estimated using a simplified version of (5)

$$
f_{\text {approx }}=\frac{68}{D \sqrt{\varepsilon_{r}}}\left(\frac{D}{2 t}+3.45\right) .
$$

As explained in the introduction, a feed of the antenna is also an important factor that can be used to enhance the bandwidth of the structure. Here a simple microstrip was used as the feed. The microstrip can excite successfully the lowest TE mode for this resonator.

The analytical approach based on the cavity resonator mode can be successfully used to analyze simple arrangements such as the cylindrical resonator on a ground plane, but for structures such as the "C" shape resonator an exact analytical solution will be far more difficult to derive. To understand better the behavior and performance of the new proposed DRA, two numerical methods have been employed. The three-dimensional model of the "C" shaped resonator mounted on top of a microstrip line on a FR-4 substrate was built and solved using two full-wave solvers, one using a finite element method (FEM) and the other one using a finite integral method (FIT). The FEM has solved the problem in frequency domain, whereas for the FIT solver the same problem was formulated in time domain. The FEM solver uses a tetrahedral mesh that is iterated at frequency of $5 \mathrm{GHz}$ until an error of less than $1 \%$ at the input port has been achieved. This mesh is then used for a discrete frequency sweep between 0.5 and $5 \mathrm{GHz}$; hence, the problem is solved in 50 discrete points in this bandwidth. The FIT solver uses a rectangular mesh which is adapted until the error between successive solutions is less than $1 \%$. The final FIT simulation using the last adapted mesh is done until accuracy better than $-30 \mathrm{~dB}$ is obtained. As the code solves the problem in time domain, this accuracy refers, in practice, to the steady state reached after the initial Gaussian pulse has passed through the structure being simulated. This value is in fact the accuracy of the frequencydomain signals that are calculated by Fourier transformation of the time signals. The two solutions are compared in terms of magnitude of return loss (S11) of the structure.

The structure used for the numerical study had the following dimensions: for the resonator diameter $D=28 \mathrm{~mm}$, thickness $t=2 \mathrm{~mm}$ and a length of the flat side $W=19.6 \mathrm{~mm}$. The microstrip had a width of $1.8 \mathrm{~mm}$ and substrate thickness was $1 \mathrm{~mm}$. The relative permittivity of the substrate was 4.9. For this geometry, the characteristic impedance of the microstrip is $50 \Omega$. The relative permittivity of the dielectric resonator was considered to be 80 , but values as high as 100 and 120 were also simulated and similar results were obtained. For these values the resonance of the first $\mathrm{TE}_{01 \delta}$ mode calculated using (6) was $\mathrm{f}_{\mathrm{TE} 01 \delta}=2.83 \mathrm{GHz}$. This value corresponds to a full cylindrical resonator, not to the "C" shape resonator. As the volume of new shape resonator is smaller than a full cylinder, one would expect the resonance of the $\mathrm{TE}_{01 \delta}$ mode to be at a slightly lower frequency. From the solution of the FIT solver, the first resonant frequency with an open stub of $L=13.45 \mathrm{~mm}$ from the end of the resonator was calculated to be $2.39 \mathrm{GHz}$, and a similar result was obtained form the FEM solution. It is interesting to note that a much better approximation of the $\mathrm{TE}_{01 \delta}$ mode resonant frequency can be obtained if the value calculated above with the approximate expression (6) is multiplied by the ratio of the volume of a full cylindrical resonator to the volume of the " $C$ " shape resonator. In this case, this ratio is 0.9 ; thus, the approximate frequency $\mathrm{f}_{\mathrm{TE} 01 \delta}=2.54 \mathrm{GHz}$, which is much closer to the numerically calculated value. The electric field vector plot at $2.39 \mathrm{GHz}$ is shown in Fig. 3 and confirms the existence of the mode $\mathrm{TE}_{01 \delta}$. In fact the first resonance of this structure can be anywhere in between 2.3 to $2.7 \mathrm{GHz}$, since it can be tuned by the length of the open microstrip stub, as explained in the next 


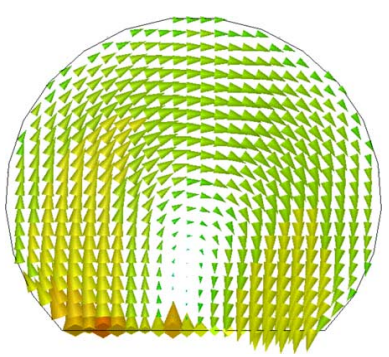

Fig. 3. Vector plot of the electric filed at $2.39 \mathrm{GHz}$ confirming the existence of the $\mathrm{TE}_{01 \delta}$ mode.

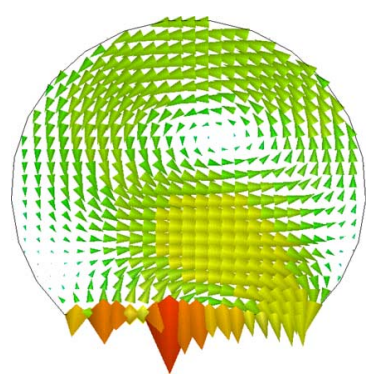

Fig. 4. Vector plot of the electric filed at $2.75 \mathrm{GHz}$ confirming the existence of the $\mathrm{HEM}_{12 \delta}$ mode.

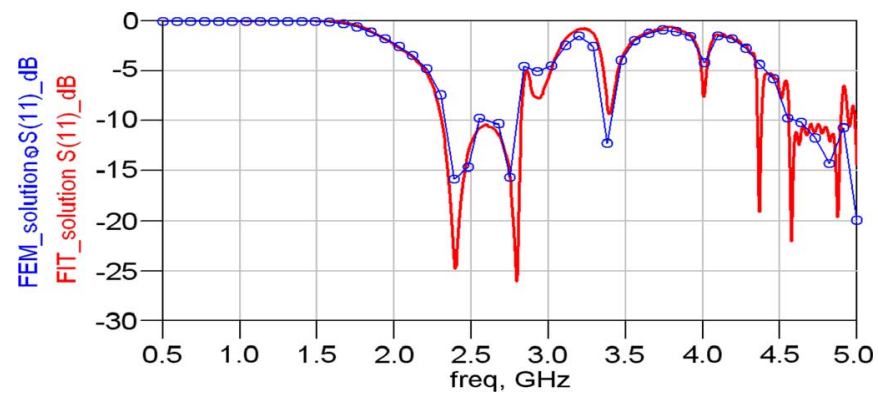

Fig. 5. Comparison of the magnitude of the calculated return loss obtained from FIT and FEM simulations.

section of this paper which deals with the characteristics of the "C" shaped DRA.

The next closest resonant mode existent in this resonator structure is a hybrid electromagnetic mode, namely $\mathrm{HEM}_{12 \delta}$. The resonant frequency of this mode is $2.75 \mathrm{GHz}$, and its existence is confirmed by the simulation data. Fig. 4 depicts the field distribution within the resonator when the $\mathrm{HEM}_{12 \delta}$ is excited.

Without any experimental data available, the only way of confirming the results obtained was through a cross check between numerical solutions obtained using different methods, in this case the time domain (FIT) and frequency-domain (FEM) solvers. The results have been compared in terms of the magnitude of the return loss (see Fig. 5).

Both methods give quite similar results - as can be seen in Fig. 5-but there are some small discrepancies at the higher range of the frequency domain. Those differences may be mainly attributed to the discrete nature of the frequency domain solution. The FEM solver calculates the field at discrete preset positions and then interpolates linearly between them, whereas the FIT solver applies a more continuous representation throughout the space in time domain and then-through a Fourier transformation-calculates the frequency response.

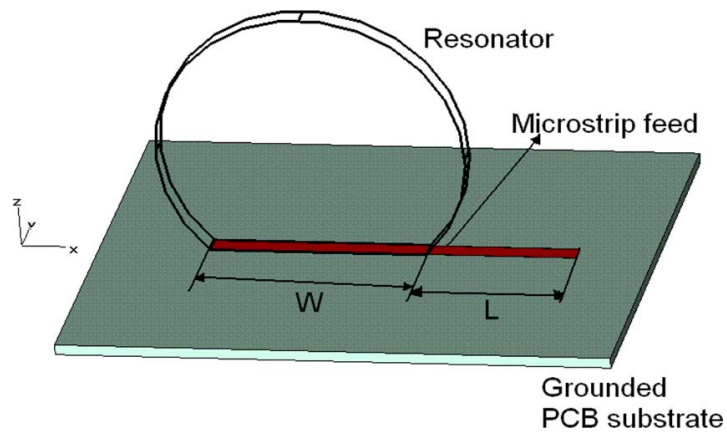

Fig. 6. DRA mounted on top of a microstrip feed on a grounded substrate ( $W$-length of the flat side of the resonator, $L$-length of the open stub).

As a result, some of the very sharp and narrow resonances, as observed above $4.2 \mathrm{GHz}$, may be missed by the FEM solver simply because not enough points in the domain of interest have been selected.

The size of the FEM mesh is related to the frequency at which the problem is solved, hence smaller (finer) meshes are required for higher frequencies as the size of the FEM elements is normally selected in proportion to the wavelength. In this study the solution obtained from the FEM method was first calculated at $5 \mathrm{GHz}$, the highest in the band of interest. Once generated, the solver used the same mesh to calculate the field solution at several discrete points that were chosen in the bandwidth of interest. The number of solutions can be freely selected and in this case 50 discrete points were used which was guided by the time taken to compute the full solution. Probably a better fit between the two solutions would have been obtained if more points were considered; however, overall both solvers gave sufficiently similar results in the bandwidth of interest $(0.5-4 \mathrm{GHz})$ to boost confidence in the results obtained, although it is appreciated that the final confirmation can only be provided by relevant measurements.

\section{ANTENNA CHARACTERISTICS}

The simulated antenna had a resonator with the dimensions described in the previous section. The resonator was mounted with the flat side over the microstrip line as sketched in Fig. 6. A standard 1-mm-thick FR4 PCB was used as a grounded substrate and a $50-\Omega$ microstrip line was used to feed the resonator.

The size of the ground was $50 \times 40 \mathrm{~mm}$. One of the advantages of the DRA antennas is the fact that they cannot be easily detuned, as the displacement currents that appear in the dielectric body are not influenced by metal structures that can exist in the near field of the radiator. One of the implications of this property is that the ground plane of this antenna has little or no influence on its behavior, unlike other electrically small antennas (F-inverted, microstrip patch or chip antenna) that are heavily influenced by their ground planes. Three different models with different size ground planes $-70 \times 50 \mathrm{~mm}$, $50 \times 40 \mathrm{~mm}$, and $40 \times 20 \mathrm{~mm}$-were simulated and no effect on the return loss of the antenna was observed.

It should be noted that the direct coupling from a microstrip line into the resonator could be weak but because of the high value of the dielectric constant of the resonator this does not create a problem in the case of the proposed arrangement.

The existence of the two close resonant modes yields the wide bandwidth observed for this antenna. The first resonant mode $\mathrm{TE}_{01 \delta}$ has an optimum coupling into the resonator when 


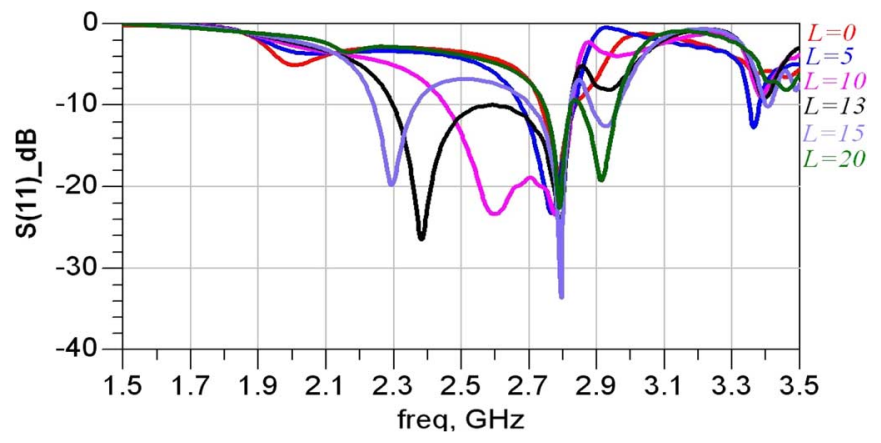

Fig. 7. Magnitude of $\mathrm{S} 11$ for different lengths of the open stub (from $L=0$ to $L=20 \mathrm{~mm}$ ).
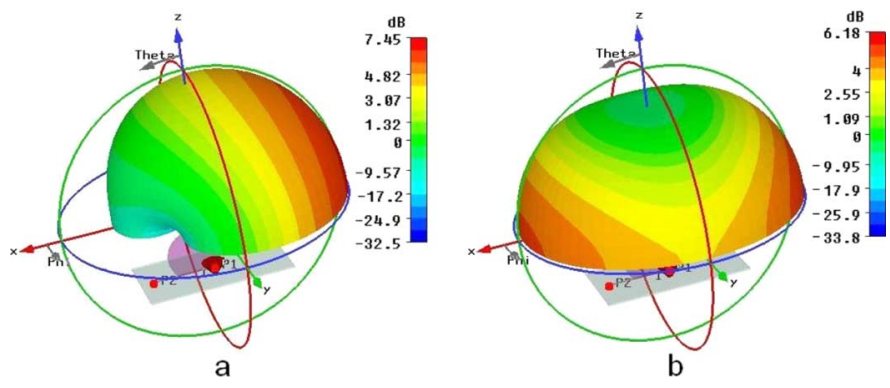

Fig. 8. Radiation patterns of the DRA. (a) $\mathrm{TE}_{01 \delta}$ resonant mode at $2.39 \mathrm{GHz}$ and (b) $\mathrm{HEM}_{12 \delta}$ resonant mode at $2.75 \mathrm{GHz}$.

the magnetic field perpendicular to its circular face has a maximum. This maximum can be achieved when the length of the microstrip line exciting the mode is equal to $\lambda_{g} / 2$, where $\lambda_{g}$ is the wavelength of the guided wave along the microstrip line. The total length of the line is $W+L$, where $W=19.6 \mathrm{~mm}$ as mentioned earlier and $L$ is the length of the open stub from the edge of the resonator. This length can be used to tune the position in frequency domain of the $\mathrm{TE}_{01 \delta}$ mode with respect to the $\mathrm{HEM}_{12 \delta}$ mode which is fixed for this structure at $2.75 \mathrm{GHz}$. The $\mathrm{HEM}_{12 \delta}$ resonant frequency depends only on the size and material properties of the dielectric resonator. It can be noticed from Fig. 7 that maximum bandwidth of approximately $500 \mathrm{MHz}$ may be achieved with $L=13 \mathrm{~mm}$ for the open stub. For this length, the total is $L+W=32.6 \mathrm{~mm}$. The half wavelength at $2.39 \mathrm{GHz}$ on the microstrip wave guide structure is $32.8 \mathrm{~mm}$, which matches nicely the resonance found by the numerical solver.

From the far-field distribution point of view, the two resonant modes existent in the antenna have different radiation patterns. This might normally be considered detrimental because the polarization of the antenna changes, but from the hand-held wireless perspective this aspect is secondary to the bandwidth and overall size of the antenna as the space in such devices is not at premium. From the plots of the radiation patterns in Fig. 8, it can be observed that at a lower frequency, when the $\mathrm{TE}_{01 \delta} \delta$ mode is excited, the main lobe is in negative $x$ axis direction, whereas at higher frequencies, when the $\mathrm{HEM}_{12 \delta}$ mode is dominant, the pattern becomes more symmetrical with its main lobe in $x-y$ plane. In both cases, the calculated radiation efficiency of the DRA is very high (close to unity) as in this structure the only losses are due to the microstrip ohmic losses. The calculated gains are 7.45 and $6.18 \mathrm{~dB}$ for the first and the second resonant modes, respectively.

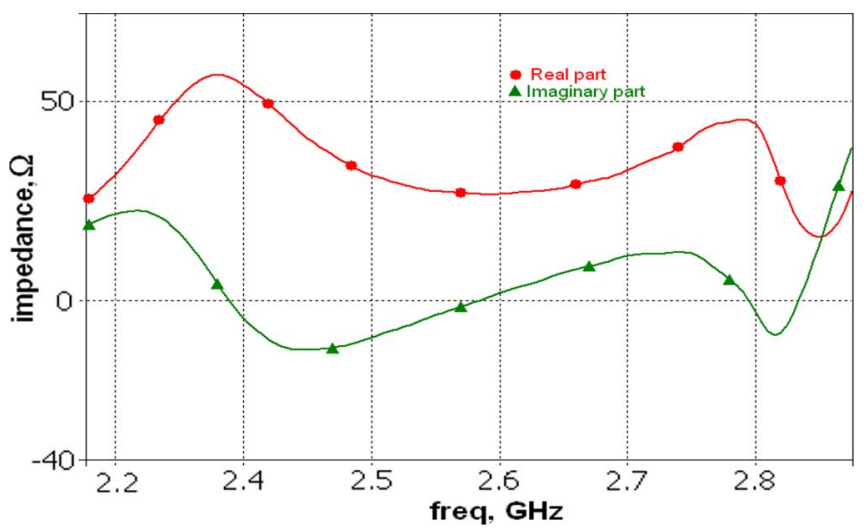

Fig. 9. Calculated input impedance for the thin "C" DRA.

The input impedance of the DRA depicted in Fig. 9 swings around $50 \Omega$ for the real part and $0 \Omega$ for the imaginary part, respectively, in the bandwidth of interest $(2.3-2.8 \mathrm{GHz})$ which will provide acceptable impedance matching.

\section{CONClusion}

In this work, a novel "C" shaped dielectric resonator antenna has been presented and its performance analyzed through numerical simulations. As the resonator does not use any regularly shaped structures (cylindrical or rectangular), the usually adopted analytical approach based on the cavity resonator model was not available to facilitate understanding or predict the performance of the new resonator. Instead, two numerical methods (FEM and FIT) were employed to solve the electromagnetic design problem. The analysis of the results has shown that the DRA based on a high-dielectric material can be used at cellular bands. Such structures would have good potential for electrically small antennas, as they are compact, provide wideband, are easy to feed, and have very high efficiency. Another major advantage for hand-held applications is the detuning insensitivity of this type of antennas. The simulation results reveal that the proposed structure can be tuned to have in excess of $500 \mathrm{MHz}$ of bandwidth at cellular frequency with very high radiation efficiency.

\section{REFERENCES}

[1] S. A. Long, M. W. McAllister, and L. C. Shen, "The resonant cylindrical dielectric cavity antenna," IEEE Trans. Antennas Propag., vol. 31, pp. 406-412, May 1983.

[2] K. W. Leung, K. Y. Chow, K. M. Luk, and E. K. N. Yung, "Low-profilecircular disk DR antenna of very high permittivity excited by a microstripline," Electron. Lett., vol. 33, no. 12, pp. 1004-1005, Jun. 1997.

[3] S. Brebels, J. Ryckaert, B. Come, S. Donnay, W. De Raedt, E. Beyne, and R. P. Mertens, "SOP integration and codesign of antennas," IEEE Trans. Adv. Packag., vol. 27, no. 2, pp. 341-351, May 2004.

[4] R. K. Mongia et al., "Theoretical and experimental investigation of rectangular dielectric resonator antennas," IEEE Trans. Antennas Propag., vol. 45, no. 9, pp. 1348-1356, 1997.

[5] M. D. Rotaru, Y. Y. Lim, H. Kuruveetil, Y. Rui, A. P. Popov, and C. P. Chua, "Implementation of package integrated antenna with embedded front end for bluetooth applications," IEEE Trans. Adv. Packag., 2008, accepted for publication.

[6] K.-M. Luk and K.-W. Leung, Dielectric Resonator Antennas. Stevenage, U.K.: Research Studies Press, Ltd., 2002.

[7] R. K. Mongia and P. H. Bhartia, "Dielectric resonator antennas-A review and general design relations for resonant frequency and bandwidth," Int. J. Microw. Millimetre-Wave Comput. Aided Eng., vol. 4, no. 3, pp. 230-247, 1994.

[8] D. Kajfez and P. Guillon, Dielectric Resonators. Norwood, MA: Artech House, 1986. 